\author{
Jennifer L. Zantinge, Hung \\ Chang Huang ${ }^{1}$, and Kuo Joan \\ Cheng $^{2}$ \\ Alberta Agriculture Food and \\ Rural Development \\ Lacombe, Alberta, Canada \\ ${ }^{1}$ Agriculture and Agri-Food \\ Canada \\ Lethbridge, Alberta, Canada \\ ${ }^{2}$ Institute of BioAgricultural \\ Sciences \\ Taipei, Taiwan
}

For reprints of this or any other article, contact Reprints@BioTechniques.com

\section{Bacteriophage Gene Targeting Vectors Generated by Transplacement}

BioTechniques 33:806-812 (October 2002)

\begin{abstract}
A rate-determining step in gene targeting is the generation of the targeting vector. We have developed bacteriophage gene targeting vectorology, which shortens the timeline of targeting vector construction. Using retro-recombination screening, we can rapidly isolate targeting vectors from an embryonic stem cell genomic library via integrative and excisive recombination. We have demonstrated that recombination can be used to introduce specific point mutations or unique restriction sites into gene targeting vectors via transplacement. Using the cholinelethanolamine kinase $\alpha$ and $\beta$ genes as models, we demonstrate that transplacement can also be used to introduce specifically a neo resistance cassette into a gene targeting phage. In our experience, the $\lambda T K$ gene targeting system offers considerable flexibility and efficiency in TV construction, which makes generating multiple vectors in one week's time possible.
\end{abstract}

\section{INTRODUCTION}

With the estimated discovery of more than 35000 genes via the genome projects, there is considerable interest in determining the function of these genes in the emerging era of functional genomics. Here there is a need to speed up the process of targeting vector generation and to develop technologies for the generation of subtle mutations beyond the gene knockout. We used bacteriophage $\lambda$ as an efficient vector for scaffolding and transferring genomic alterations to embryonic stem cells via gene targeting. We have previously developed phage-plasmid recombination to direct modification cassettes to specific sites within phage targeting vectors via double crossover (5). Small regions of homology are used to direct modification cassettes to specific sites within the recombination-proficient gene targeting phages $(5,7)$. A supressor tRNA gene supF, positioned within the homologous region, acts as a positive selectable marker for recombination by suppressing amber mutations in $\lambda \mathrm{TK}$. A negative marker gene, gam, is placed outside the region of homology to enrich for double-crossover recombinants by selectively killing single-crossover recombinants via spi selection $(2,4)$.

We have also established new methods that use the sequential integrative and excisive recombination of plasmids. Retro-recombination screening allows the rapid purification of phage targeting vectors from an embryonic stem cell targeting vector library in $\lambda \mathrm{TK}$ (7), following the homologous integration of a $\sup F$-bearing recombination plasmid. As the region of homology is duplicated on integration and can revert under relaxed conditions, we incorporated gam into the recombination plasmid to spi select phages that have reverted to their original configuration $(6,7)$. Using retro-recombination screening, we isolated specific targeting vector phages in 2-3 days to serve as templates for the rapid completion of targeting vector construction. An alternative method, called transplacement mutagenesis, uses an integrative and excisive recombination strategy to transfer point mutations to target phages using a recombination plasmid interface $(6,8)$. Following the excision of the plasmid, a mutation is transferred precisely in approximately $50 \%$ of condensatants. We routinely introduce unique restriction sites into $\lambda \mathrm{TK}$ phages for the subsequent introduction of modification cassettes.

In theory, transplacement can also be used to introduce larger changes, including sequence additions, provided that sufficient regions of homology are included. Accordingly, we have investigated whether transplacement could be used as a strategy for introducing $n e o^{R}$ selection cassettes using integrative and excisive recombination (Figure 1). Using the mouse choline/ethanolamine kinase genes $\alpha$ and $\beta$ (CK/EK $\alpha$ and $\beta)$ (1) as a template, we demonstrate that retro-recombination screening and transplacement can be combined to insert unique restriction sites as targeting vectors are plaque-purified. We also show how modification cassettes can be introduced into targeting vectors via transplacement.

\section{MATERIALS AND METHODS}

\section{Bacterial Strains, Recombination Plasmid Constructs, and Bacterio- phages}

The $E$. coli strains, $\lambda \mathrm{TK}$ embryonic stem cell library, and recombination plasmid $\pi \mathrm{AN} \gamma$ have been described previously $(6,7)$. Recombination plasmid $\alpha$ sx $\pi$ AN $\gamma$ (Figure 2A) was created by ligating two genomic PCR fragments that surround the seventh exon of $\mathrm{CK} / \mathrm{EK} \alpha$ into $\pi \mathrm{AN} \gamma$ to introduce two novel restriction sites (SalI and XhoI) into the recombination probe sequence. A 247-bp (+19 non-homologous region) 5'EcoRI-XhoI fragment was generated by genomic PCR using forward (5'-AAAGAATTCCAGATAGTAGCTGAG-3') and reverse (5'-AAACTCGAGTCGACCTACAAGAGATTTGG-3') primers. Similarly, a 213-bp (+16) 3' XhoI-BamHI fragment was generated by genomic PCR using forward (5'AAACTCGAGAATATCTTATTGTTGGAAG- $\left.3^{\prime}\right)$ and reverse (5'-AAAGGATCCTCAGGGACAAACA-3') primers. Its derivative, $\alpha$ sxneo $\pi \mathrm{AN} \gamma$, was generated by inserting a XhoI cassette of pol2:neo $o^{R}$ in opposite transcriptional orientation to the CK/EK $\alpha$ gene. For $\beta$ sx $\pi \mathrm{AN} \gamma$, a 194-bp (+18) 5' EcoRI- 
XhoI fragment was generated by genomic PCR using forward (5'-AAAGAATTCCCAGGACTGGAGCCACC$\left.3^{\prime}\right)$ and reverse $\left(5^{\prime}\right.$-AAACTCGAGTCGACTACCCCTGTCCCGT-3') primers. Similarly, a 198-bp (+15) 3' XhoIBamHI fragment was generated by genomic PCR using forward (5'-AAACTCGAGGAGGAGGGGCTGTC-3') and reverse (5'-AAAGGATCCTCACGGGACAAACGCTC-3') primers. Its derivative, $\beta$ sxneo $\pi \mathrm{AN} \gamma$ (Figure $2 \mathrm{~A}$ ) was generated by inserting the pol2: $n e o^{R}$ cassette downstream of the CK/ EK $\beta$ initiation codon, in opposite transcriptional orientation to the gene.

\section{Retro-Recombination Screening and Transplacement Mutagenesis}

Retro-recombination screening was performed as previously described (8). Following retro-recombination screening, isolated plaques from the $\alpha \operatorname{sx} \pi \mathrm{AN} \gamma$ and $\beta \mathrm{sx} \pi \mathrm{AN} \gamma$ screen were analyzed by PCR and restriction endonuclease diges- tion to identify clones that underwent transplacement and retained the engineered XhoI and SalI restriction sites. Both $\mathrm{CK} / \mathrm{EK} \alpha$ and $\beta$ phage eluates were screened by PCR using primers lying outside the original regions of homology that had been used in the recombination plasmids ( $\alpha$ forward, $5^{\prime}$-TTCTTCACCATGGAGCCG-3', and $\alpha$ reverse, $5^{\prime}-\mathrm{G}-$ AGTTCCAGAAGAGCTAA-3'; $\beta$ forward, 5'-CTCAGTCAGTAAGAGCAGA-3', and $\beta$ reverse, 5'-GGAGGAGATCAAGGTCTTA-3'). Positive phages were also screened for the presence of a novel SalI site using digestion of the corresponding PCR products. Positive clones were subsequently restriction mapped to confirm the position of the introduced $\mathrm{SalI} / \mathrm{XhoI}$ restriction sites.

Phage-plasmid recombination using $\alpha \mathrm{sx} \pi \mathrm{AN} \gamma-$ and $\beta$ sx $\pi \mathrm{AN} \gamma$-positive phage isolates and their corresponding $n e o^{R}$ recombination plasmids were used to investigate whether the $n e o^{R}$ cassette could be transplaced into a specific phage using integrative and excisive re-

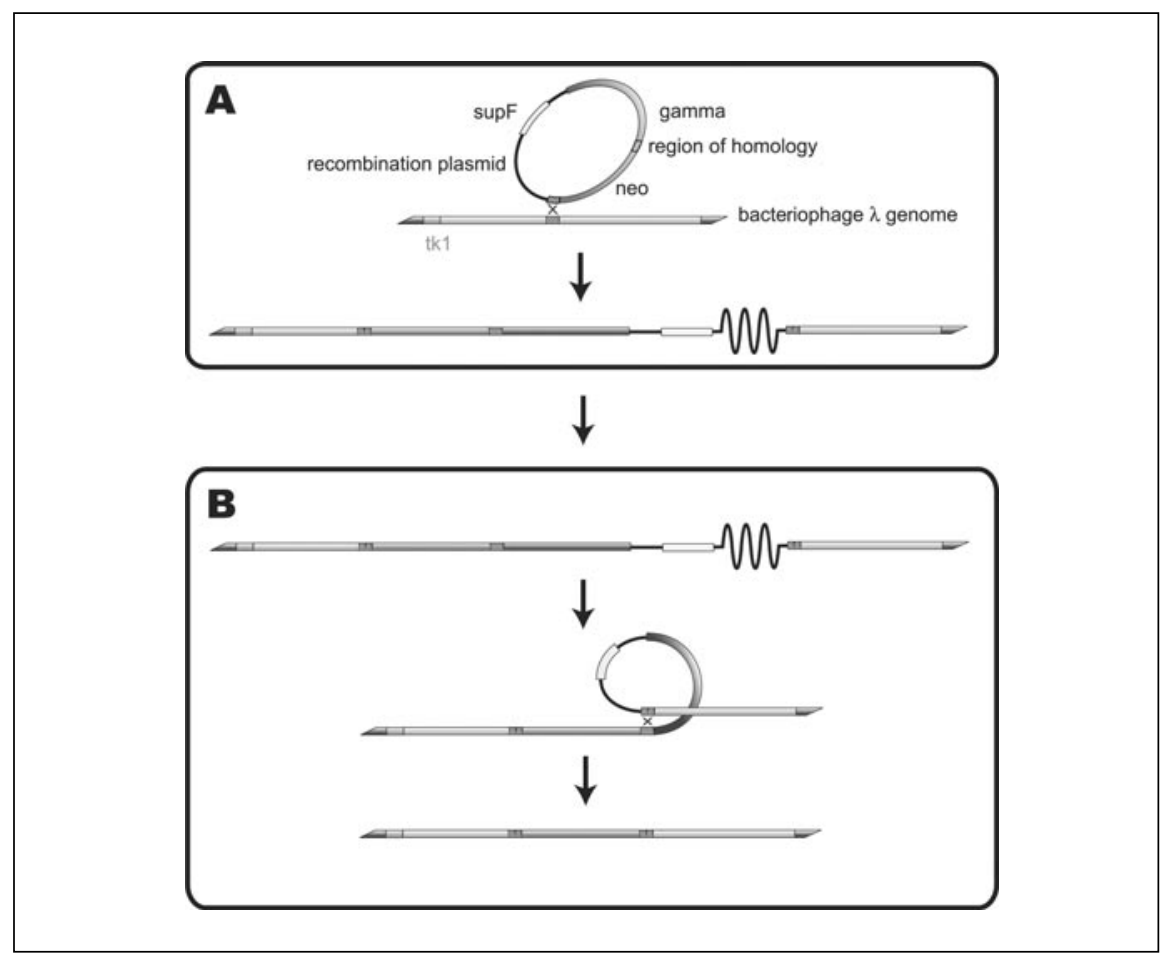

Figure 1. Transplacement of selectable marker genes via phage plasmid recombination. A two-step procedure is used to transfer selectable markers from split homology recombination plasmids to targeting vectors in $\lambda \mathrm{TK}$. (A) A recombination plasmid, bearing supF and gam markers and a split homologous region with a centered $n e o^{R}$ cassette, is integrated into $\lambda \mathrm{TK}$ by a single-crossover recombination event and selected using supF. (B) Following the relaxation of supF selection, phages that underwent condensation are enriched for by spi selection against $\mathrm{gam}$. When excisional recombination occurs contra-laterally to the integration site (approximately $50 \%$ of the time), the $n e o^{R}$ cassette is left behind in the target phage. Note that the plasmid and phage are not drawn to scale. 
combination. The $\lambda$ TKCK/EK $\alpha$ or $\beta$ phages $\left(1 \times 10^{4} \mathrm{PFU}\right)$ were passaged over MC1061[p3] bearing osxneo $\pi-$ AN $\gamma$ and $\beta$ sxneo $\pi \mathrm{AN} \gamma$, respectively. Supernatant phages were collected the next day and used to infect LG75 for the selection of single-crossover recombinants, which were then cored and eluted in SM buffer. Phages having undergone condensation were isolated by spi selection on P2392. Plaques were screened by hybridization using a neo probe to identify phage clones that retained neo. DNA from such clones was isolated in mini-scale (3) and digested to confirm the transplacement of $n e o^{R}$ and the identity of the resulting $\lambda$ TKCK/EK $\alpha$ or $\beta$ targeting vectors.

\section{RESULTS AND DISCUSSION}

Both retro-recombination screening and transplacement take advantage of a cycle of integrative and excisive recombination, through which a recombination plasmid $\pi \mathrm{AN} \gamma$ that bears a short region that is homologous to sequences in a target phage is integrated and excised by a single-crossover recombination event $(5,7,8)$. Based on their similarity, it is theoretically possible to combine both processes to isolate specific $\lambda \mathrm{TK}$ phage-bearing unique restriction sites. To test whether restriction sites could be transplaced into $\lambda$ TK gene-targeting phages via retro-recombination screening, two recombination plasmids were constructed using homology derived from genomic sequences of the CK/EK $\alpha$ or $\beta$ genes (Figure 2). The probe sequences within $\alpha \mathrm{sx} \pi \mathrm{AN} \gamma$ and $\beta \mathrm{sx} \pi$ AN $\gamma$ contained 460 and 392 bp of homology surrounding exon 7 and exon 1 of $\mathrm{CK} / \mathrm{EK} \alpha$ or $\beta$, respectively. Each plasmid contained SalI and XhoI sites that were approximately central within the disrupted probe homology region
( $\alpha, 247$ and $213 \mathrm{bp}$, and $\beta, 194$ and 198 $\mathrm{bp})$. These plasmids and their corresponding controls, $\alpha \pi \mathrm{AN} \gamma$ and $\beta \pi \mathrm{AN} \gamma$ (data not shown), which did not contain the introduced restriction sites were used to screen $4 \times 10^{7}$ PFU of the $\lambda$ TK embryonic stem cell library via retrorecombination screening.

Because centered mutations within transplacement vectors disrupt the homology, we determined that larger regions of homology were needed. Table 1 shows that the homology break introduced in $\alpha s x \pi \mathrm{AN} \gamma$ and $\beta$ sx $\pi \mathrm{AN} \gamma$ significantly reduced the frequency of plasmid-phage recombination. The control probe plasmids gave a robust recovery of clones. As expected, considerably fewer clones (approximately 2fold) were recovered for $\beta \mathrm{CK} / \mathrm{EK}$, based on the smaller region of homology that was used. However, in both cases, a marked difference was observed using the transplacement plasmids. For 
Table 1. Phage-Plasmid Recombination Screening Frequencies of CK/EK $\alpha$ and $\beta$ Probe Plasmids with and without Centered $\mathrm{SalI}$ and $\mathrm{XhoI}$ Restriction Sites

\begin{tabular}{|c|c|c|c|}
\hline Vector & Phage Titera & No. Blue Plaques & $\begin{array}{c}\text { Recombination } \\
\text { Screening Frequencyb }\end{array}$ \\
\hline$\alpha \pi \mathrm{AN} \gamma$ & $6 \times 10^{9}$ & $2 \times 10^{3}$ & $3.3 \times 10^{-7}$ \\
\hline$\alpha s \times \pi A N \gamma$ & $2.4 \times 10^{10}$ & $9 \times 10^{2}$ & $3.8 \times 10^{-8}$ \\
\hline$\beta \pi \mathrm{AN} \gamma$ & $2.5 \times 10^{9}$ & $2.3 \times 10^{2}$ & $9.2 \times 10^{-8}$ \\
\hline$\beta s x \pi A N \gamma$ & $3.3 \times 10^{9}$ & $2 \times 10^{2}$ & $6.1 \times 10^{-9}$ \\
\hline \multicolumn{4}{|c|}{$\begin{array}{l}\text { Each plasmid harbored in } \mathrm{MC} 1061[\mathrm{p} 3] \text { was used to screen the } \lambda T K \text { phage library } \\
\text { (approximately } 4 \times 10^{8} \mathrm{PFU} / \mathrm{mL} \text { ). Processed lysates were titered on LE392, after } \\
\text { which } 10^{9}-10^{10} \mathrm{PFU} \text { were used to screen for co-integrants on LG75 (blue } \\
\text { plaques). } \\
\text { aTiter on LE392 expressed as PFU/mL. } \\
\text { bRecombination screening frequencies were determined for each individual data } \\
\text { set using the formula of number of blue plaques/phage titer. }\end{array}$} \\
\hline
\end{tabular}

the larger $\alpha$ sxCK/EK probe, a 9-fold reduction in the recovery of recombinants was observed over wild-type $\alpha \mathrm{CK} / \mathrm{EK}$, whereas a 15-fold reduction was ob- served for the smaller $\beta$ sxCK/EK probe over the wild-type $\beta C K / E K$. Nonetheless, a significant number of recombinants (at least 20) were observed for each of these probe plasmids, which indicates the possibility that considerably smaller regions of balanced homology might be used for successful retrorecombination screening using transplacement probe plasmids.

Following isolation, 10 individual recombinant clones were passaged sequentially over the sup $F^{+}$host LE392 and its P2 lysogen, P2392, to allow both condensation and spi selection. In agreement with our previous data (7), the condensation frequency was very high $(10 \%-30 \%)$ for each recombinant because of the long lengths of homology used (data not shown). In contrast, when oligonucleotide-based regions of homology (approximately $50 \mathrm{bp}$ ) are used, this frequency drops by one order of magnitude (6). Whereas it might be expected that condensation frequency would occur less frequently in clones bearing split homology regions, this was not observed because of the short- 
ening of contiguous homology. Instead, comparable frequencies or greater frequencies were observed for the split homology plasmid over the control. These results suggest that when one is using larger homology regions, cis recombination frequencies are less responsive to contiguous homology than trans.

After condensation of the $\alpha \operatorname{sx} \pi \mathrm{AN} \gamma$ and $\beta \mathrm{sx} \pi \mathrm{AN} \gamma$ clones, DNA from individual plaques was examined for the transplacement of SalI and XhoI sites. Table 2 indicates that, among the small number of clones that were examined, $50 \%$ or more transplaced these sites. Surprisingly, despite the unbalanced homology in the $\alpha$ sx probe (247 and $213 \mathrm{bp}$ ), the transplacement frequency remained at $50 \%$. This is in sharp contrast to our previous experiments using oligonucleotide-based homologies, where subtle differences in length of homology or GC content can have a tremendous effect on the transplacement frequency (unpublished results). This result reiterates the idea that a threshold of homology is sufficient for cis recombination and that the choice of sides for excisive recombination may be stochastic.

For both the CK/EK $\alpha$ and $\beta$ genes, multiple, distinct $\lambda \mathrm{TK}$ clones were isolated that represented different regions of their respective loci. Figure 2B displays the restriction maps of the contigs isolated using retro-recombination transplacement. In each clone, both SalI and XhoI sites were transferred to their respective region of homology. The introduction of these rare restriction sites helped to determine rapidly the boundaries of each clone isolated from the library, facilitating the selection of a centralized clone for the completion of the targeting vector and flanking clones for the isolation of a flanking probe.

For both the CK/EK $\alpha$ and $\beta$ clones, the introduced $X h o$ site was not unique and confounded the completion of the targeting vectors via the introduction of an XhoI cassette of $n e o^{R}$. Accordingly, we investigated whether a $n e o^{R}$ selection cassette could also be introduced using transplacement. The $n e o^{R_{\text {-con- }}}$ taining homology plasmids, $\alpha s x-$ neo $\pi \mathrm{AN} \gamma$ and $\beta$ sxneo $\pi \mathrm{AN} \gamma$ harbored in MC1061[p3], were used as a host to shuttle $n e o^{R}$ into $\lambda$ TK $\alpha$ and $\beta$ CK/EK phages. Single phages from the $\alpha$ and $\beta$ series were selected as substrates, based on the criteria that they were relatively small (approximately $12 \mathrm{~kb}$ ) and centered within the contig (Figure 2B).

In a total of nine analyses, the transplacement of $n e o^{R}$ occurred approximately $50 \%$ of the time (Table 2 ). As expected, restriction analysis of representative clones demonstrated that the $n e o^{R}$ gene was introduced into the seventh and first exons of CK/EK $\alpha$ and $\beta$,

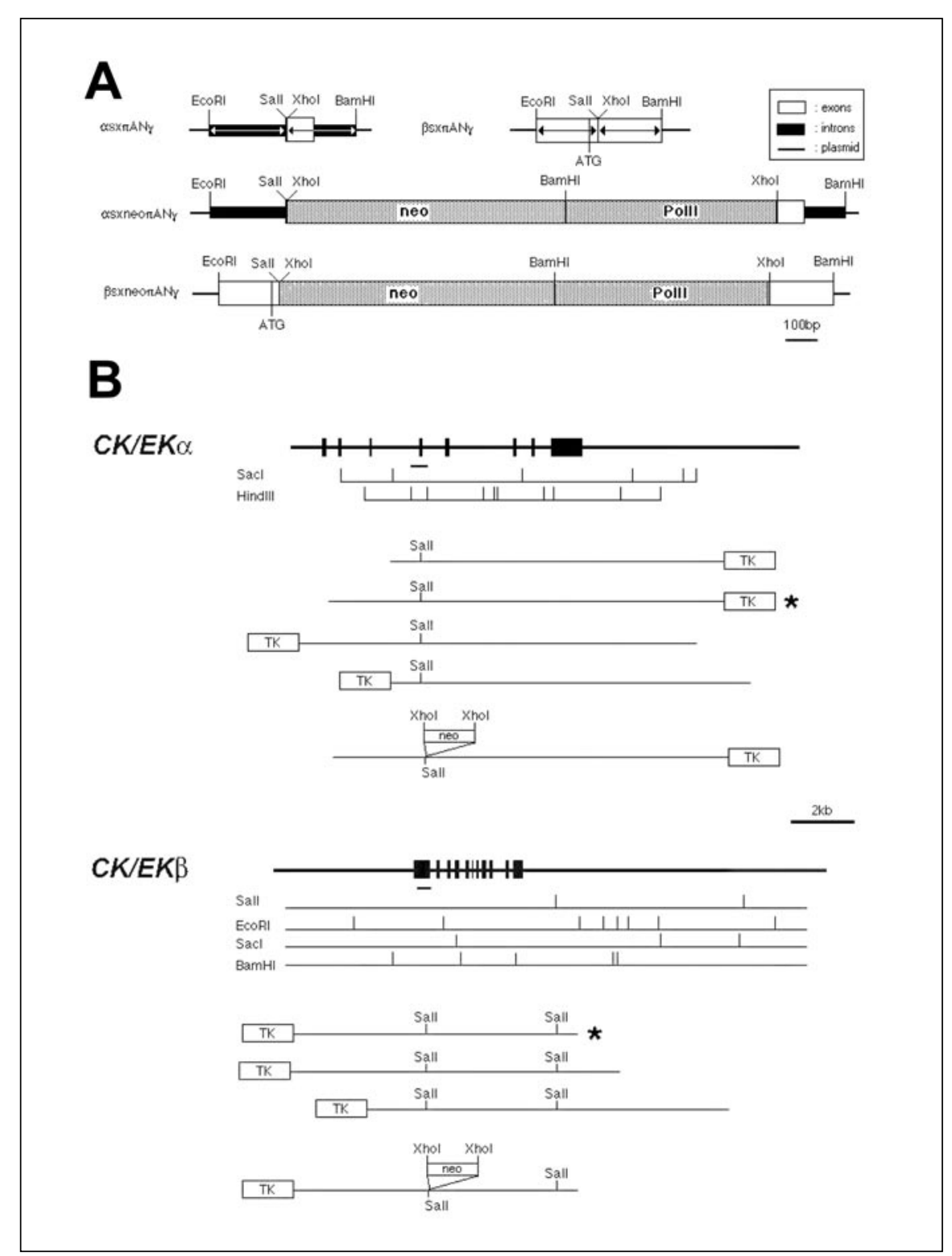

Figure 2. CK/EK recombination plasmids and $\lambda$ TK clones following transplacement. (A) $\alpha \pi \mathrm{AN} \gamma$ and $\beta \pi \mathrm{AN} \gamma$ derivatives were generated by subcloning genomic PCR fragments derived from the $\alpha$ and $\beta$ $\mathrm{CK} / \mathrm{EK}$ genes into $\pi \mathrm{AN} \gamma$. $\alpha \mathrm{sx} \pi \mathrm{AN} \gamma$ and $\beta \mathrm{sx} \pi \mathrm{AN} \gamma$ were created by ligating two genomic PCR fragments to introduce centered SalI/XhoI sites into these sequences. Accordingly, the $5^{\prime}$ and $3^{\prime}$ regions of homology used for transplacement are denoted by divergent arrows. Their derivatives, $\alpha$ sxneo $\pi \mathrm{AN} \gamma$ and $\beta$ sx$\pi \mathrm{AN} \gamma$, were generated by the insertion of a XhoI pol2:neo ${ }^{R}$ cassette in the opposite transcriptional orientation. (B) Contigs of CK/EK $\alpha$ and $\beta$ clones were generated from the $\lambda \mathrm{TK}$ clones that were isolated by retro-recombination screening transplacement. The bold line of each represents the gene structure. The dash beneath displays the probe homology region used. Following the gene structure diagram are diagnostic restriction maps of each locus. The individual $\lambda \mathrm{TK}$ clones that were isolated are portrayed. In these clones, SalI and XhoI sites were deposited into the middle of the region of homology. The asterisks represent clones that were used to generate targeting vectors using $n e o^{R}$ transplacement. For these clones, their respective neo insertions are indicated at the bottom of both the CK/EK $\alpha$ and $\beta$ panels. 


\section{Short Technical Reports}

Table 2. Transplacement Frequencies of Restriction Sites or $n e o^{R}$ Selectable Marker after Integrative and Excisive Recombination Using CK/EK $\alpha$ and $\beta$ Probe Plasmids

\begin{tabular}{|cccc|}
\hline Vector & Total Screened & Positive Clones & Frequency \\
\hline$\alpha s \times \pi \mathrm{AN} \gamma$ & 8 & 4 & 0.5 \\
$\beta s \times \pi \mathrm{AN} \gamma$ & 12 & 7 & 0.58 \\
$\alpha \mathrm{sxneo} \pi \mathrm{AN} \gamma$ & 87 & 64 & \\
& 93 & 40 & 0.54 \\
& 101 & 49 & \\
& 33 & 15 & \\
$\beta$ ssxneo $\pi \mathrm{AN} \gamma$ & 50 & 161 & 0.6 \\
& 236 & 101 & \\
& 115 & 69 & \\
Single-crossover recombinants were grown under relaxed conditions in LE392. \\
Phages from this liquid lysis culture were passaged on P2392 to isolate phages \\
that had undergone a condensation event. Resulting phages were analyzed for \\
the presence of novel Sall/Xhol sites by restriction digestion and neo $R$ by hy- \\
bridization.
\end{tabular}

respectively (Figure 2B). Like the retrorecombination screening transplacement experiments, the transfer of $n e o^{R}$ was as efficient as transferring restriction sites. Unlike the $\beta$ experiments in which the regions of homology were balanced, no significant difference was detected with the $\alpha$ experiments when the regions of homology regions were slightly unbalanced, which again indicated that a certain low threshold of homology is sufficient for efficient recombination in cis.

In summary, we have demonstrated that transplacement provides a powerful, genetic approach to isolate and modify rapidly targeting vectors from a $\lambda \mathrm{TK}$ gene targeting library. Unlike double-crossover techniques previously described in which deleterious bacterial selection markers are deposited into targeting vectors $(5,9)$ using transplacement, it is now possible to keep the extraneous selectable marker outside the region of homology. In addition, selection markers are recycled in transplacement, thus allowing an indefinite number of rapid sequential modifications such as the introduction of loxP sites after $n e o^{R}$ transplacement. Using this genetic approach, targeting vector construction is not labor intensive and allows multiple vectors to be constructed at one time. Thus, in the next generation of structure/function gene targeting studies, batteries of targeting vectors with different subtle mutations may be generated in synchrony. No doubt, in the post-genome era where mouse genomic sequences will be readily available, complicated targeting vectors will be constructed in a week using transplacement.

\section{REFERENCES}

1.Aoyama, C., N. Yamazaki, H. Terada, and K. Ishidate. 2000. Structure and characterization of the genes for murine choline/ethanolamine kinase isozymes $\alpha$ and $\beta$. J. Lipid Res. 41:452-464.

2.Haggard-Ljungquist, E., V. Barreiro, R. Calendar, D.M. Kurnit, and H. Cheng. 1989. The P2 phage old gene: sequence, transcription and translational control. Gene 85:25-33.

3.Sambrook, J., E.F. Fritsch, and T. Maniatis. 1989. Molecular Cloning: A Laboratory Manual, 2nd ed. CSH Laboratory Press, Cold Spring Harbor, NY.

4.Shen, P. and H.V. Huang. 1986. Homologous recombination in Escherichia coli: dependence on substrate length and homology. Genetics 112:441-457.

5.Tsuzuki, T. and D.E. Rancourt. 1998. Embryonic stem cell gene targeting using bacteriophage $\lambda$ vectors generated by phage-plasmid recombination. Nucleic Acids Res. 26: 988-993.

6.Unger, M.W.T., S.Y. Liu, and DE. Rancourt. 1999. Transplacement mutagenesis: a novel in situ mutagenesis system using phage-plasmid recombination. Nucleic Acids Res. 27:14801484.
7.Woltjen, K., G. Bain, and D.E. Rancourt. 2000. Retro-recombination screening of a mouse embryonic stem cell genomic library. Nucleic Acids Res. 28:E41.

8.Woltjen, K., M.W. Unger, and D.E. Rancourt. 2002. Transplacement mutagenesis. A recombination-based in situ mutagenesis protocol. Methods Mol. Biol. 182:189-207.

9.Zhang, P., M.Z. Li, and S.J. Elledge. 2002 Towards genetic genome projects: genomic library screening and gene TV construction in a single step. Nat. Genet. 30:31-39.

We thank Brad Thomas for drawing Figure 1. C.A. was supported by The Japanese Society for the Promotion of Science for Young Scientists. K.W. was funded by studentships from the AHFMR and NSERC. F.C.M. was funded by post-doctoral fellowships from the AHFMR and MRC. This work was supported in part by Special Coordination Funds for Promoting Science and Technology (to K.I.) from MonbuKagaKu-sho of Japan and by infrastructure grants (to D.E.R.) from the Alberta Cancer Board and CIHR. Financial support (to K.I.) from Sankyo Co. Ltd. is also appreciated. D.E.R. is an AHFMR scholar. K.I. and D.E.R. are senior authors on this manuscript. Address correspondence to Dr. Derrick E. Rancourt, $374 \mathrm{HMRB}, 3330 \mathrm{Hospi-}$ tal Dr. N.W., Calgary, Alberta T2N4N1, Canada.e-mail: rancourt@ucalgary.ca

Received 1 May 2002; accepted 8 July 2002.

C. Aoyama, K. Woltjen' ${ }^{1}$, F.C. Mansergh ${ }^{1}, K$. Ishidate, and D.E. Rancourt ${ }^{1}$ Tokyo Medical and Dental University Chiyodaku, Tokyo ${ }^{1}$ University of Calgary Calgary, Alberta, Canada

For reprints of this or any other article, contact Reprints@BioTechniques.com 